\title{
Underwater Image Enhancement using Color Balance and Fusion
}

\author{
T Yasoda Vijaya Lakshmi ${ }^{1} \mid$ A Lilly ${ }^{1} \mid$ Md. V J Shareef ${ }^{1} \mid$ V Rajesh ${ }^{1} \mid$ Y Appa Rao ${ }^{1}$ \\ ${ }^{1}$ Department of ECE, VSM College of Engineering, Ramachandrapuram, AP, India.
}

To Cite this Article

T Yasoda Vijaya Lakshmi, A Lilly, Md. V J Shareef, V Rajesh and Y Appa Rao, “Underwater Image Enhancement using Color Balance and Fusion", International Journal for Modern Trends in Science and Technology, Vol. 06, Issue 06, June 2020, pp.:43-45; https://doi.org/10.46501/IJMTST060610

\section{Article Info}

Received on 28-April-2020, Revised on 20-May-2020, Accepted on 25-May-2020, Published on 04-June-2020.

\section{ABSTRACT}

We have introduced an elective way to deal with upgrade the pictures caught submerged and corrupted because of the medium dispersing and assimilation. Our technique is a solitary picture approach that doesn't require particular equipment or information about the submerged conditions or scene structure. It expands on the mixing of two pictures that are straightforwardly gotten from a shading redressed and white-adjusted rendition of the first debased picture. The two pictures to combination, just as their related weight maps, are characterized to advance the exchange of edges and shading complexity to the yield picture. To stay away from that the sharp weight map changes make ancient rarities in the low recurrence parts of the reproduced picture, we additionally adjust a multi scale combination technique. Our broad subjective and quantitative assessment uncovers that our upgraded pictures and recordings are described by better exposedness of the dull districts, improved worldwide difference, and edges sharpness. Our approval likewise demonstrates that our calculation is sensibly free of the camera settings, and improves the exactness of a few picture preparing applications.

KEYWORDS: assimilation, shading complexity.

Copyright (C) 2014-2020 International Journal for Modern Trends in Science and Technology

DOI: https://doi.org/10.46501/IJMTST060610

\section{INTRODUCTION}

Submerged condition offers numerous uncommon attractions, for example, marine creatures and angles, stunning scene, and secretive wrecks. Unique in relation to basic pictures, submerged pictures experience the ill effects of poor perceivability coming about because of the constriction of the engendered light, fundamentally because of retention and dissipating impacts. The assimilation considerably lessens the light vitality, while the dissipating causes alters in the light engendering course. They bring about foggy appearance and difference debasement, making far off items dim. For all intents and purposes, in like manner ocean water pictures, the items a ways off of in excess of 10 meters are practically unperceivable, and the hues are blurred on the grounds that their creating frequencies are sliced by the water profundity.

Conversely, this paper acquaints a novel methodology with expel the cloudiness in submerged pictures dependent on a solitary picture caught with a regular camera. Our approach expands on the combination of numerous information sources, yet infers the two contributions to consolidate by rectifying the difference and by honing a white-adjusted form of a solitary local information picture. The white 
adjusting stage targets expelling the shading cast actuated by submerged light dissipating, to deliver a characteristic appearance of the sub-ocean pictures. The multi-scale execution of the combination procedure brings about a relic free mixing.

\section{BLOCK DIAGRAM AND SOFTWARE COMPONENTS}

\section{Block Diagram}

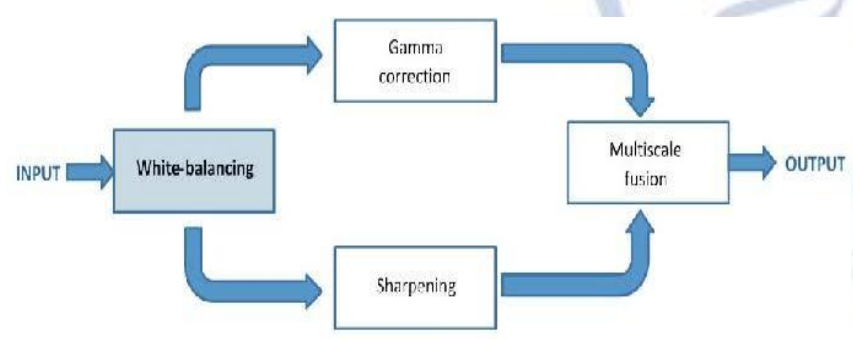

Fig: Block diagram

Since the shading remedy is basic in submerged, we initially apply our white adjusting procedure to the first picture. This progression targets upgrading the picture appearance by disposing of undesirable shading throws brought about by different illuminants.

In water further than $30 \mathrm{ft}$, white adjusting experiences observable impacts since the consumed hues are hard to be recuperated. Accordingly, to acquire our first info we play out a gamma remedy of the white adjusted picture rendition. Gamma remedy targets adjusting the worldwide differentiation and is applicable since, by and large, white adjusted submerged pictures will in general show up excessively splendid. This revision expands the distinction between darker/lighter districts at the expense of lost subtleties in the under-over-uncovered areas.

To make up for this misfortune, we determine a second information that compares to a honed variant of the white adjusted picture. In this way, we follow the unsharp covering rule, as in we mix an obscured or unsharp (here Gaussian separated) adaptation of the picture with the picture to hone.

The weight maps are utilized during mixing so that pixels with a high weight esteem are progressively spoken to in the last picture. They are subsequently characterized dependent on various nearby picture quality or saliency measurements.

By autonomously utilizing a combination procedure at each scale level, the potential ancient rarities because of the sharp advances of the weight maps are limited. Multi-scale combination is persuaded by the human visual framework, which is touchy to sharp advances showing up in smooth picture designs, while being substantially less delicate to varieties/antiques happening on edges and surfaces (veiling wonder). Curiously, an ongoing work has indicated that the multiscale procedure can be approximated by a computationally proficient furthermore, outwardly wonderful single-scale system. This single scale guess should be energized when multifaceted nature is an issue, since it likewise transforms the multi goals process into a spatially restricted system.

\section{Software Components we used in this project is: MATLAB}

\section{WORKING}

The overview of this method are two images, is derived from the (too pale) white balanced image, using Gamma correction and edge sharpening, respectively. Those two images are then used as inputs of the fusion process, which derives normalized weight maps and blends the inputs based on a multi-scale process. The multi-scale fusion approach is here exemplified with only three levels of the Laplacian and Gaussian pyramids.
Green Channel

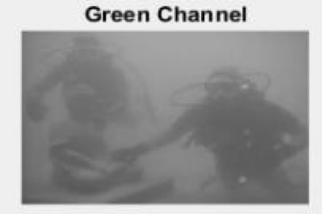

Original Image

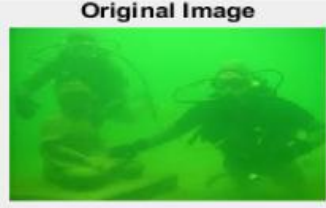

II. Gamma Corrected

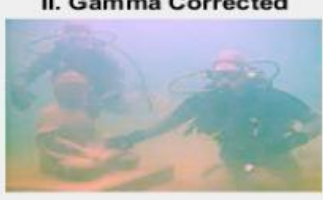

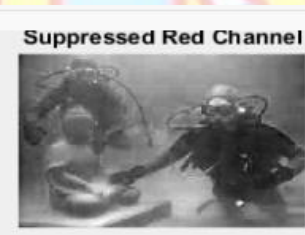

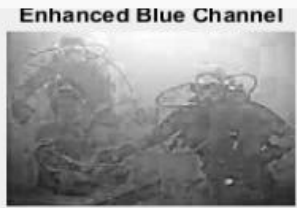

After White balance

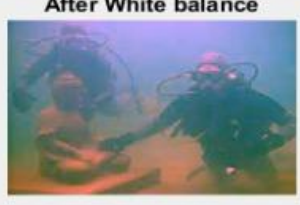

I. White Balance

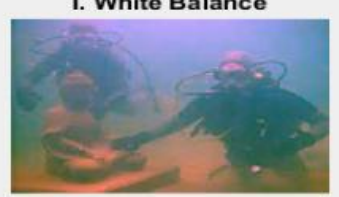

III. Sharpened

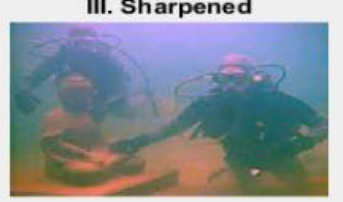




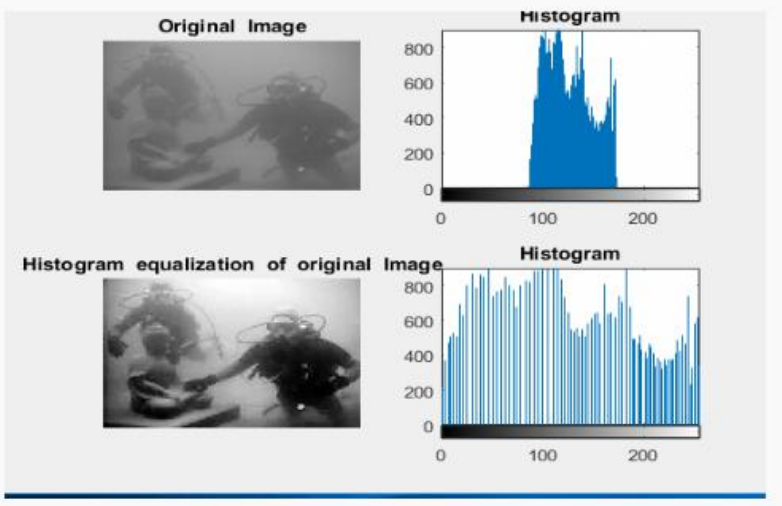

Fig : Working Flow

\section{Applications and Conclusion}

\section{Applications}

1. Underwater imaging

2. Seafloor survey

3. Underwater pipeline monitoring

4. Maintenance of Underwater structures

5. Biological Monitoring

\section{v. Conclusion}

We have presented an alternative approach to enhance underwater videos and images. Our strategy builds on the fusion principle and does not require additional information than the single original image. We have shown in our experiments that our approach is able to enhance a wide range of underwater images (e.g. different cameras, depths, light conditions) with high accuracy, being able to recover important faded features and edges. Moreover, for the first time, we demonstrate the utility and relevance of the proposed image enhancement technique for several challenging underwater computer vision applications.

\section{REFERENCES}

[1]M. D. Kocak, F. R. Dalgleish, M. F. Caimi, and Y. Y. Schechner, "A focus on recent developments and trends in underwater imaging," Marine Technol. Soc. J., vol. 42, no. 1 , pp. 52-67, 2008

[2]R. Schettini and S. Corchs, "Underwater image processing: state of the art of restoration and image enhancement methods," EURASIP J. Adv.Signal Process., vol. 2010, Dec.2010, Art. No. 746052.

[3]R. Fattal, "Single image dehazing," in Proc. ACM SIGGRAPH, Aug. 2008, Art. no. 72.

[4]C. O. Ancuti and C. Ancuti, "Single image dehazing by multi-scale fusion," IEEE Trans. Image Process., vol. 22, no. 8, pp. 3271-3282 Aug. 2013.

[5]J. Y. Chiang and Y.-C. Chen,"Underwater image enhancement by wavelength compensation and dehazing," IEEE Trans. Image Process., vol. 21, no. 4, pp. 1756-1769, Apr. 2012. 